\title{
COMPARISON OF PERTURBED DIRAC OPERATORS
}

\author{
JEFFREY FOX AND PETER HASKELL
}

(Communicated by Palle E. T. Jorgensen)

\begin{abstract}
This paper extends the index theory of perturbed Dirac operators to a collection of noncompact even-dimensional manifolds that includes both complete and incomplete examples. The index formulas are topological in nature. They can involve a compactly supported standard index form as well as a form associated with a Toeplitz pairing on a hypersurface.
\end{abstract}

\section{INTRODUCTION}

When a Dirac operator is perturbed by an order zero term that is invertible off a compact set, it is frequently the case that the perturbed operator is Fredholm and has its index given by a topological formula. The paper of Callias [Ca] has motivated a number of proofs of such results on complete noncompact odddimensional manifolds $[\mathrm{A} 2, \mathrm{BM}, \mathrm{Bu}, \mathrm{Ga}, \mathrm{R}]$ as well as a proof for complete noncompact even-dimensional manifolds $[\mathrm{Bu}]$. The way in which such results depend on topological aspects of the given data suggests that there should be a common index theory that includes perturbed Dirac operators on manifolds that are complete or incomplete and of any dimension. In $[\mathrm{FH}]$ we identified Fredholm perturbed Dirac operators on the incomplete smooth subsets of odd-dimensional manifolds with isolated conical singularities. We also established an index formula for such operators that is completely analogous to the formula arising on complete odddimensional manifolds. In the present paper we extend this area of index theory to even-dimensional manifolds, both complete and incomplete. Again the incomplete examples are smooth subsets of manifolds with isolated conical singularities.

The index formula for a perturbed Dirac operator on an even-dimensional manifold is a consequence of the Atiyah-Singer index theorem, applied to an operator on a compact manifold constructed from the data defining the perturbed Dirac operator. Thus this index formula is topological in nature. In some examples it can be viewed as the integral of an odd index form over a hypersurface. In general the index formula can be regarded as containing contributions of two types: an integral of a compactly supported standard index form and the hypersurface term. Heat kernel techniques provide a way to exhibit both terms explicitly. (In contrast the

Received by the editors October 24, 1994.

1991 Mathematics Subject Classification. Primary 58G10.

Key words and phrases. Perturbed Dirac operator.

Jeffrey Fox's work was supported by the National Science Foundation.

Peter Haskell's work was supported by the National Science Foundation under Grant No. DMS-9204275. 
index formula for a perturbed Dirac operator on an odd-dimensional manifold can be regarded entirely as a hypersurface term: the other term vanishes for dimension reasons.)

The basic technical tool in this paper is the observation that an index matching argument of [GuHi] extends to our setting. This reasoning reveals explicitly the common ground shared by the complete and incomplete cases. In its conclusion the index matching theorem is similar to a relative index theorem [GLa]. Each says that only the behavior on a certain compact set matters. However the hypotheses of these theorems are different. The relative index theorem permits more general operators but assumes they are identified by an isometry outside the compact sets (which can differ). The index matching theorem requires that the operators be identified by an isometry of the compact sets and that each operator have a wellbehaved perturbation that is invertible off the compact sets. However it requires no similarity between the complements of the compact sets. In a remark at the end we note that the index matching argument relates adiabatic limits of indices of perturbed Dirac operators to adiabatic limits of reduced eta invariants.

\section{Perturbed Dirac operators}

This section defines the perturbed Dirac operators we are interested in, establishes the properties they have that are important to us, and provides examples of perturbed Dirac operators with these properties.

Let $M$ be an even-dimensional spin manifold. Let $\mathcal{D}$ be the Dirac operator from sections of the positive spinor bundle $S_{+}$to sections of the negative spinor bundle $S_{-}$. Let $\mathcal{D}^{*}$ be its formal adjoint. Later we address questions of domain for these operators. For now one can assume that the domain of each operator consists of the set of smooth compactly supported sections.

Let $E_{0}$ and $E_{1}$ be a pair of auxiliary Hermitian vector bundles on $M$. Le $\mathcal{A}$ be a smooth vector bundle map from $E_{0}$ to $E_{1}$. Assume that there is a compact subset of $M$ off of which $\mathcal{A}$ is pointwise invertible. Let $\mathcal{A}^{*}$ be the pointwise adjoint from $E_{1}$ to $E_{0}$. By slight abuse of notation let $\mathcal{A}$ and $\mathcal{A}^{*}$ denote also the associated linear maps between spaces of sections of $E_{0}$ and $E_{1}$. Again we will clarify domains when necesary.

Putting metric connections and trivial Clifford actions on $E_{0}$ and $E_{1}$, we can form tensor product connections on $S_{ \pm} \otimes E_{i}$. For $i \in\{0,1\}$ let $D$ denote the resulting Dirac operator from sections of $S_{+} \otimes E_{i}$ to sections of $S_{-} \otimes E_{i}$. Define $D^{*}$ similarly. Let $A$ be the operator from sections of $S_{ \pm} \otimes E_{0}$ to sections of $S_{ \pm} \otimes E_{1}$ defined by the tensor product of the identity operator with $\mathcal{A}$. Define $A^{*}$ similarly.

Definition 1.1. By a perturbed Dirac operator we mean an operator

$$
D_{A}=\left(\begin{array}{cc}
A & D^{*} \\
D & -A^{*}
\end{array}\right) \text {. }
$$

This operator maps sections of $S_{+} \otimes E_{0} \oplus S_{-} \otimes E_{1}$ to sections of $S_{+} \otimes E_{1} \oplus S_{-} \otimes E_{0}$.

Assume $M$ is a complete noncompact manifold. A perturbed Dirac operator, defined originally on smooth compactly supported sections, has a unique closed extension as an operator on $L^{2}$ sections [Cf]. We denote this closed operator $D_{A}$. The reasoning in [A1], which is adapted from [GLa], provides conditions under 
which $D_{A}$ is Fredholm. For instance, let

$$
D_{A}^{\prime}=\left(\begin{array}{cc}
0 & D_{A}^{*} \\
D_{A} & 0
\end{array}\right)
$$

and let

$$
P=\left(\begin{array}{cc}
D^{*} D & 0 \\
0 & D D^{*}
\end{array}\right)
$$

Write

$$
\left(D_{A}^{\prime}\right)^{2}=\left(\begin{array}{cc}
P & 0 \\
0 & P
\end{array}\right)+R_{A}
$$

Lemma 1.2 ([A1]). Assume $M$ is complete. If $R_{A}$ is a vector bundle map for which there exists a positive constant $k$ such that off some compact set $R_{A} \geq k$ pointwise, then $D_{A}$ is Fredholm.

One can verify that if $M$ is a complete manifold with an end equal to a linearly expanding warped product and if $\mathcal{A}$ is radially constant on the end, then the hypotheses of the above lemma are satisfied. An explicit description of such $M$ and $\mathcal{A}$ is as follows.

Example 1.3. $M$ is divided by a submanifold $N$ into two parts: a compact manifold with boundary, where $N$ is the boundary; and, for some positive $p$, an end $M_{e}$ equal to $\{(r, n): p \leq r, n \in N\}$ with Riemannian metric $d r \otimes d r+r^{2} g_{N}$. Here $g_{N}$ is a Riemannian metric on $N$. M is the union of these two pieces modulo identification of each point $n$ in the boundary of the first piece with the corresponding point $(p, n)$ in the second piece.

For $i \in\{0,1\}$ the restriction to $M_{e}$ of $E_{i}$ behaves as the product of $[p, \infty)$ with a Hermitian bundle $\widetilde{E_{i}} \rightarrow N$. Thus there are fixed isomorphisms of Hermitian vector bundles $\left.E_{i}\right|_{M_{e}} \cong[p, \infty) \times \widetilde{E_{i}}$. There is an invertible vector bundle map $\widetilde{\mathcal{A}}: \widetilde{E_{0}} \rightarrow \widetilde{E_{1}}$. The above isomorphisms identify the restriction of $\mathcal{A}$ to any $\left\{r_{0}\right\} \times N$ with $\widetilde{\mathcal{A}}$. The connection on each $E_{i}$ is flat in the radial direction.

Definition 1.4. We say that a perturbed Dirac operator $D_{A}$ realizes its limiting index if the family of perturbed Dirac operators $D_{t A}$, parametrized by $t \in[1, \infty)$, is a family of Fredholm operators with constant index.

Lemma 1.5. Suppose that $A$ is bounded and that the perturbed Dirac operator $D_{A}$ on a complete manifold $M$ is Fredholm because the associated $R_{A}$ satisfies the hypotheses of Lemma 1.2. Then $D_{A}$ realizes its limiting index.

Proof. Because the term in $R_{t A}$ that involves $t^{2}$ is pointwise positive off a compact set, $R_{t A} \geq R_{A}$ for all $t \geq 1$. Thus $D_{t A}$ is Fredholm for all $t \geq 1$. Because $A$ is bounded, when we view $D_{t A}$ as a family of bounded operators from $\operatorname{domain}(D)$ to $L^{2}\left(S_{+} \otimes E_{1} \oplus S_{-} \otimes E_{0}\right)$, this family is norm continuous.

Remark 1.6. Write $D_{A}^{\prime}=d+a$, where $d$ consists of all entries involving $D$ or $D^{*}$ and $a$ consists of all entries involving $A$ or $A^{*}$. Then $R_{A}=d a+a d+a^{2}$, and $R_{t A}=t\left(d a+a d+t a^{2}\right)$.

Remark 1.7. When $A$ is unbounded, the most common assumptions leading to a Fredholm perturbed Dirac operator on a complete noncompact manifold $M$ involve 
assuming that, with respect to the distance from some fixed point in $M$, the growth of the pointwise minimum eigenvalue of $|a|$ dominates the growth of $d a+a d$. Here $a$ and $d$ are as in Remark 1.6. (See [Ga] for an example of such assumptions.) Then off some compact set, $R_{t A} \geq R_{A}$ for all $t \geq 1$. Domain $\left(D_{t A}\right)$ is independent of $t$ for $t \geq 1$, and $A$ is a bounded operator from $\operatorname{domain}\left(D_{A}\right)$ to

$$
L^{2}\left(S_{+} \otimes E_{1} \oplus S_{-} \otimes E_{0}\right) .
$$

It follows that $D_{t A}$ is a norm-continuous family of Fredholm operators from $\operatorname{domain}\left(D_{A}\right)$ to $L^{2}\left(S_{+} \otimes E_{1} \oplus S_{-} \otimes E_{0}\right)$, and so $D_{A}$ realizes its limiting index.

Example 1.8. Let $M$ be the set of smooth points of a compact space with isolated metrically conical singularities. $M$ is an incomplete smooth Riemannian manifold that is divided by a submanifold $N$ into two parts: a compact manifold with boundary, where $N$ is the boundary; and a finite-length metric cone over $N$. This cone equals, for some positive $p,\{(r, n): 0<r \leq p, n \in N\}$ with Riemannian metric $d r \otimes d r+r^{2} g_{N}$. Here $g_{N}$ is a Riemannian metric on $N$. We denote this cone by $C_{0, p}(N) . M$ is the union of these two pieces modulo identification of each point $(p, n)$ in the cone with the corresponding point $n$ in the boundary of the first piece.

Let $\widetilde{S}$ be the spinor bundle over $N$. For $i \in\{0,1\}$ let $\widetilde{E_{i}}$ denote the restriction of $E_{i}$ to $N$. In the language described in Example 1.3, assume that all structures on the $\widetilde{E}_{i}$ are radially constant on the cone and that $A$ is of the form $r^{-1}$ times an operator that is radially constant on the cone. Denote the restriction of $A$ to $N$ by $\widetilde{A}$. Let $\widetilde{D_{i}}$ be the Dirac operator on sections of $\widetilde{S} \otimes \widetilde{E_{i}}$. Making allowance for the difference in dimensions, one can reason as in [L] to show that over the cone $D_{A}$ is unitarily equivalent to

$$
\partial / \partial r+r^{-1}\left(\begin{array}{cc}
\widetilde{D_{0}} & \widetilde{A^{*}} \\
\widetilde{A} & -\widetilde{D_{1}}
\end{array}\right)
$$

as an operator on the Hilbert space of $L^{2}$ functions on $(0, p]$ with values in $L^{2}\left(\widetilde{S} \otimes \widetilde{E_{0}} \oplus \widetilde{S} \otimes \widetilde{E_{1}}\right)$. (Of course the operator is only densely defined. For the moment one can assume it is defined on smooth compactly supported functions with values in smooth sections.) We use the following notation:

$$
T_{A}=\left(\begin{array}{cc}
\widetilde{D_{0}} & \widetilde{A^{*}} \\
\widetilde{A} & -\widetilde{D_{1}}
\end{array}\right) .
$$

Assumption 1.9. In discussing perturbed Dirac operators $D_{A}$ on manifolds with isolated conical singularities, we always assume that $A$ has been chosen so that the spectrum of $T_{A}$ has empty intersection with $(-1 / 2,1 / 2)$.

Lemma 1.10 ([BS1]). Under Assumption $1.9 D_{A}$, defined on smooth compactly supported sections, has a unique closed extension, which we will also denote $D_{A}$, as an operator on $L^{2}$ sections. This extension is Fredholm.

Lemma 1.11. For $A$ as described in Example 1.8, $\left\{s: D_{s A}\right.$ satisfies Assumption $1.9\}$ contains an interval of the form $[k, \infty)$.

Proof. The proof is analogous to that given in the odd-dimensional case in [FH]. In short, in $T_{s A}^{2}$ the only term that is not guaranteed to be positive is linear in $s$, whereas there is a positive term that is multiplied by $s^{2}$. 
Lemma 1.12. For $A$ as described in Example 1.8, $\left\{s: D_{s A}\right.$ realizes its limiting index $\}$ contains an interval of the form $\left[k_{1}, \infty\right)$.

Proof. Lemmas 1.10 and 1.11 show that there is such an interval of values of $s$ for which $D_{s A}$ is Fredholm. Reasoning like that used in the proof of Lemma 1.11 shows that, for large enough $s, T_{s A}+T_{s A}^{2}$ has a positive lower bound. Thus for large enough $s_{1}$ and $s_{2}$ each of $T_{s_{1} A}+T_{s_{1} A}^{2}$ and $T_{s_{2} A}+T_{s_{2} A}^{2}$ is bounded relative to the other. It follows that for large enough $s \operatorname{domain}\left(D_{s A}\right)$ is independent of $s$. The existence of the positive lower bound for $T_{s A}+T_{s A}^{2}$ also implies that $A$ is a bounded operator from the common domain of the $D_{s A}$ to the space of $L^{2}$ sections of the appropriate bundle. Thus, for large enough $s, D_{s A}$ is a norm-continuous family of bounded Fredholm operators from the common domain of the $D_{s A}$ to $L^{2}$.

Remark 1.13. Associated with a perturbed Dirac operator $D_{A}$ on a noncompact manifold $M$ is a perturbed Dirac operator $\widehat{D_{\hat{A}}}$ on a compact manifold $\widehat{M}$ that is constructed as follows. This construction is a generalization of a construction apppearing in $[\mathrm{GuHi}]$.

Let $K$ be the compact subset of $M$ on which $A$ is not invertible. Let $\overline{M_{0}}$ be a neighborhood of $K$ that is a smooth manifold with boundary. Denote by $M_{0}$ the interior of $\overline{M_{0}}$. Let $\widehat{M}$ be the double of $\overline{M_{0}}$, and give $\widehat{M}$ a spin structure that agrees with that of $M$ in a neighborhood of $K$. Let $\widehat{S_{ \pm}}$denote the spinor bundles over $\widehat{M}$.

Let $\widehat{E_{0}}$ be the vector bundle over $\widehat{M}$ formed by clutching copies of $E_{0}$ over $\overline{M_{0}}$ and $\widehat{M} \backslash M_{0}$ via the identity map over the boundary of $\overline{M_{0}}$. Let $\widehat{E_{1}}$ be the vector bundle over $\widehat{M}$ formed by clutching a copy of $E_{1}$ over $\overline{M_{0}}$ with a copy of $E_{0}$ over $\widehat{M} \backslash M_{0}$ via a clutching map that is the restriction of $A^{-1}$ to the boundary of $\overline{M_{0}}$. Give $\widehat{E_{0}}$ and $\widehat{E_{1}}$ Hermitian structures and metric connections that agree with those on $E_{0}$ and $E_{1}$ over some neighborhood of $K$. Define $\widehat{A}: \widehat{S_{ \pm}} \otimes \widehat{E_{0}} \rightarrow \widehat{S_{ \pm}} \otimes \widehat{E_{1}}$ to agree with $A$ over $\overline{M_{0}}$ and with the identity on $E_{0}$ over $\widehat{M} \backslash M_{0}$. Let $\widehat{D}$ be the Dirac operator from sections of $\widehat{S_{+}} \otimes \widehat{E_{i}}$ to sections of $\widehat{S_{-}} \otimes \widehat{E_{i}}$.

$$
\widehat{D_{\hat{A}}}=\left(\begin{array}{cc}
\widehat{A} & \widehat{D}^{*} \\
\widehat{D} & -\widehat{A}^{*}
\end{array}\right)
$$

maps sections of $\widehat{S_{+}} \otimes \widehat{E_{0}} \oplus \widehat{S_{-}} \otimes \widehat{E_{1}}$ to sections of $\widehat{S_{+}} \otimes \widehat{E_{1}} \oplus \widehat{S_{-}} \otimes \widehat{E_{0}}$. Under the natural identification of the subsets $M_{0}$ of $M$ and $M_{0}$ of $\widehat{M}, D_{A}$ and $\widehat{D_{\hat{A}}}$ agree on a neighborhood of $K$.

Remark 1.14. Because $\widehat{M}$ is compact and because $\widehat{D_{\hat{A}}}$ is a first-order elliptic operator perturbed by an order zero term, $\widehat{D_{\hat{A}}}$ realizes its limiting index.

Lemma 1.15. Extend the notation used in Remark 1.6 to include the setting of Example 1.8. For each perturbed Dirac operator shown in this section to realize its limiting index there exist a compact set $C$ and a positive constant $c$ such that, for large enough $t, R_{t A} \geq c$ on the complement of $C$.

Proof. If $M$ is complete, $A$ is bounded, and $R_{A}$ satisfies the hypotheses of Lemma 1.2 , then the reasoning used to prove Lemma 1.5 proves that the inequality holds. For unbounded $A$ on complete $M$, the growth assumption and its discussion in Remark 1.7 establish the inequality. In the case of the conical singularity, let $\widetilde{a}$, 
respectively $\widetilde{d}$, denote the operator made from the entries of $T_{A}$ in which $\widetilde{A}$ or its adjoint, respectively $\widetilde{D}$ or its adjoint, appears. Then

$$
a d+d a+a^{2}=r^{-2}\left(\begin{array}{cc}
\widetilde{a}+\widetilde{a} \widetilde{d}+\widetilde{d} \widetilde{a}+\widetilde{a}^{2} & -\widetilde{a}+\widetilde{a} \widetilde{d}+\widetilde{d} \widetilde{a}+\widetilde{a}^{2}
\end{array}\right) .
$$

The conclusion follows from the observations that $\widetilde{a}^{2}$ is pointwise positive on the compact manifold $N$ and that

$$
R_{t A}=r^{-2} t\left(\begin{array}{cc}
\widetilde{a}+\widetilde{a} \widetilde{d}+\widetilde{d} \widetilde{a}+t \widetilde{a}^{2} & -\widetilde{a}+\widetilde{a} \widetilde{d}+\widetilde{d} \widetilde{a}+t \widetilde{a}^{2}
\end{array}\right) .
$$

We restate the conclusion of this lemma as an assumption to be used later.

Assumption 1.16. There exist a compact set $C$ and a positive constant $c$ such that, for large enough $t, R_{t A} \geq c$ on the complement of $C$.

\section{INDEX MATCHING}

In this section we show that the reasoning of [GuHi, §4] proves an index matching theorem for perturbed Dirac operators.

Notation 2.1. Let $D_{A}$ be a Fredholm perturbed Dirac operator on a manifold $M$. Let $E_{0}$ and $E_{1}$ be the associated auxiliary bundles. Let $X_{B}$ be a Fredholm perturbed Dirac operator on a manifold $W$. Let $F_{0}$ and $F_{1}$ be the auxiliary bundles.

Theorem 2.2. Assume that each of $D_{A}$ and $X_{B}$ realizes its limiting index and that each satisfies Assumption 1.16. Assume that there are neighborhoods $U$ of the singular set of $A$ and $V$ of the singular set of $B$ that are isometric. Assume that the isometry is covered by an isomorphism of graded Hermitian vector bundles $\left.\left.\left(S_{ \pm} \otimes E_{0,1}\right)\right|_{U} \rightarrow\left(S_{ \pm} \otimes F_{0,1}\right)\right|_{V}$ and that the associated map on sections intertwines the restrictions of $D_{A}$ and $X_{B}$. Then

$$
\operatorname{index}\left(D_{A}\right)=\operatorname{index}\left(X_{B}\right) .
$$

Proof. This proof is adapted directly from the proof of a special case given in [GuHi]. Let $f$ be the map on sections defined by the isomorphism of Hermitian vector bundles. Let $\phi$ be a nonnegative function that is identically one on the singular set of $A$ and that has support in $U$. Let $\Phi$ denote multiplication by $\phi$.

Because $D_{A}$ and $X_{B}$ realize their limiting indices, in order to show index $\left(D_{A}\right)=$ index $\left(X_{B}\right)$ it suffices to show that for large enough $t$

$$
\operatorname{index}\left(\left(\begin{array}{cc}
D_{t A} & 0 \\
0 & X_{t B}^{*}
\end{array}\right)\right)=0 .
$$

Let

$$
G_{t}=\left(\begin{array}{cc}
D_{t A} & -(t f \circ \Phi)^{*} \\
t f \circ \Phi & X_{t B}^{*}
\end{array}\right) .
$$

Because $G_{t}$ is a relatively compact perturbation of the operator in (2.3), it suffices to show that, for large enough $t, G_{t}$ is invertible. We proceed to show that, for 
large enough $t, G_{t}^{*} G_{t}$ has a positive lower bound. Analogous reasoning establishes the same property for $G_{t} G_{t}^{*}$.

Because $\phi$ has compact support, the off-diagonal entries of $G_{t}^{*} G_{t}$ are bounded operators with linear dependence on $t$.

The upper left and lower right diagonal blocks of $G_{t}^{*} G_{t}$ admit analyses that are similar to each other. We do the former explicitly. The upper left block of $G_{t}^{*} G_{t}$ is of the form

$$
P+t\left(\alpha \delta+\delta \alpha+t \alpha^{2}\right)+t^{2}\left(\Phi f^{*} f \Phi\right)
$$

where $t\left(\alpha \delta+\delta \alpha+t \alpha^{2}\right)$ is the upper left diagonal block of $R_{t A}$. The operator in (2.4) can be rewritten as

$$
P+t(\alpha \delta+\delta \alpha)+t^{2}\left(\alpha^{2}+\Phi f^{*} f \Phi\right)
$$

Because $\alpha^{2}+\Phi f^{*} f \Phi$ has a positive lower bound, Assumption 1.16 and the fact that $t^{2}$ dominates $t$ imply that the operator in (2.4) has a positive lower bound for large enough $t$.

Remark 2.6. There is an analogous index matching theorem for the perturbed Dirac operators on odd-dimensional manifolds considered in [A2,BM,Bu,Ca,FH,Ga,R].

Remark 2.7. For a perturbed Dirac operator on an even-dimensional manifold as considered here, the index matching theorem and the construction of Remark 1.13 permit one to use the Atiyah-Singer index theorem to derive an index formula for the perturbed Dirac operator.

Remark 2.8. When $E_{0}$ and $E_{1}$ are trivial, the index class for $\widehat{D_{\hat{A}}}$ is the cap product of the homology Chern character of the Dirac operator with the image under the connecting homomorphism of the cohomology Chern character of the restriction of $\mathcal{A}$ to the boundary of $\overline{M_{0}}$. The index of $D_{A}$ equals the index of the Toeplitz operator formed from the restriction of $\mathcal{A}$ to the boundary of $\overline{M_{0}}$ and the Dirac operator on this boundary.

Remark 2.9. When $M$ has conical singularities, the index formula of [BS1] applies. As in [B2, proof of Theorem 4.4], the term in the formula associated with residues of eta invariants vanishes. The remaining terms are the integral of a standard index form and a reduced eta invariant on $N$. If $D_{A}$ realizes its limiting index, then the adiabatic limit calculations of $[\mathrm{BiC}]$ provide the formula for this eta invariant term that is analogous to the boundary contribution discussed in Remark 2.8.

Remark 2.10. More generally, if $D_{A}$ realizes its limiting index and satisfies Assumption 1.16, one can use the index matching theorem to equate index $\left(D_{A}\right)$ with the index of a perturbed Dirac operator on a manifold formed by attaching a cone to the boundary of $\overline{M_{0}}$. Then Remark 2.9 applies.

\section{REFERENCES}

[A1] N. Anghel, An abstract index theorem on non-compact Riemannian manifolds, Houston J. Math. 19 (1993), 223-237. MR 94c:58193

[A2] N. Anghel, $L^{2}$-index formulae for perturbed Dirac operators, Commun. Math. Phys. 128 (1990), 77-97. MR 91b:58243 
[BiC] J.-M. Bismut and J. Cheeger, $\eta$-invariants and their adiabatic limits, J. Amer. Math. Soc. 2 (1989), 33-70. MR 89k:58269

[B1] J. Brüning, $L^{2}$-index theorems on certain complete manifolds, J. Differential Geom. 32 (1990), 491-532. MR 91h:58103

[B2] J. Brüning, On $L^{2}$-index theorems for complete manifolds of rank-one type, Duke Math. J. 66 (1992), 257-309. MR 93i:58145

[BM] J. Brüning and H. Moscovici, $L^{2}$-index for certain Dirac-Schrödinger operators, Duke Math. J. 66 (1992), 311-336. MR 93g:58142

[BS1] J. Brüning and R. Seeley, An index theorem for first order regular singular operators, Am. J. Math. 110 (1988), 659-714. MR 89k:58271

[BS2] J. Brüning and R. Seeley, The resolvent expansion for second order regular singular operators, J. Funct. Anal. 73 (1987), 369-429. MR 88g:35151

[Bu] U. Bunke, On constructions making Dirac operators invertible at infinity, preprint, 1992.

[Ca] C. Callias, Axial anomalies and index theorems on open spaces, Commun. Math. Phys. 62 (1978), 213-234. MR 80h:58045a

[Cf] P. Chernoff, Essential self-adjointness of powers of generators of hyperbolic equations, J. Funct. Anal. 12 (1973), 401-414. MR 51:6119

[FH] J. Fox and P. Haskell, Index theory for perturbed Dirac operators on manifolds with conical singularities, Proceedings of the Amer. Math. Soc. 123 (1995), 2265-2273. MR 95i:58172

[Ga] C. Gajdzinski, $L^{2}$-index for perturbed Dirac operator on odd dimensional open complete manifold, preprint, 1993.

[GLa] M. Gromov and H. B. Lawson, Positive scalar curvature and the Dirac operator on complete Riemannian manifolds, Publ. Math. I.H.E.S. 58 (1983), 83-196. MR 85g:58082

[GuHi] E. Guentner and N. Higson, A note on Toeplitz operators, preprint, 1993.

[L] M. Lesch, Deficiency indices for symmetric Dirac operators on manifolds with conic singularities, Topology 32 (1993), 611-623. MR 94e:58133

[R] J. Råde, Callias' index theorem, elliptic boundary conditions, and cutting and gluing, Commun. Math. Phys. 161 (1994), 51-61. MR 95b:58143

Mathematics Department, University of Colorado, Boulder, Colorado 80309

E-mail address: jfox@euclid.colorado.edu

Mathematics Department, Virginia Polytechnic Institute and State University, BlacksBurg, Virginia 24061

E-mail address: haskell@math.vt.edu 